\title{
ON SOME QUESTIONS IN NOETHERIAN RINGS
}

\author{
BY LANCE W. SMALL
}

Communicated by S. Smale, March 30, 1966

1. Introduction. Goldie's theorem [1] establishes (among other things) that right Noetherian rings without nilpotent ideals have (right) classical quotient rings. In the general case the author [3] found a necessary and sufficient condition that an arbitrary right Noetherian ring have a right Artinian quotient ring. Using this criterion, it can be shown that a right hereditary, right Noetherian ring has a right Artinian quotient ring [2].

In this note we present an example of:

(1) a right and left Noetherian ring with a right, but not a left quotient ring and

(2) a right and left Noetherian ring with no quotient ring on either side.

2. Notations and definitions. From now on ring means ring with unit element, and Noetherian means right and left Noetherian. $N(R)$ denotes the maximal nilpotent ideal of a Noetherian ring $R$.

Definition. If $M$ is a subset of a ring $A$, then the right annihilator of $M, r(M)$, is $\{a \in A \mid M a=0\}$. We shall write $r_{A}(M)$ if there is a possibility of confusion about the ring. The left annihilator of $M$, $l(M)$, is defined analogously.

Recall that an element $a$ in a ring $R$ is regular if $r(a)=l(a)=0$.

Definition. A right (left) ideal $I$ of a ring $R$ is essential if $I$ intersects every nonzero right (left) ideal of $R$ nontrivially.

Definition. $Z_{r}(R)=\{a \in R \mid r(a)$ is essential $\}$ is called the (right) singular ideal of $R . Z_{l}(R)$ is defined analogously.

3. The examples. Let $\boldsymbol{Z}$ denote the integers and $p \in \boldsymbol{Z}$ a prime. Define $T$ to be the ring of all two-by-two "matrices" of the form:

$$
\left(\begin{array}{ll}
a & b \\
0 & c
\end{array}\right), \quad a \in \boldsymbol{Z}, \quad b \in \boldsymbol{Z} /(p), \quad c \in \boldsymbol{Z} /(p),
$$

where addition is component-wise and multiplication is given by:

$$
\left(\begin{array}{ll}
a & b \\
0 & c
\end{array}\right)\left(\begin{array}{cc}
a^{\prime} & b^{\prime} \\
0 & c^{\prime}
\end{array}\right)=\left(\begin{array}{cc}
a a^{\prime} & a b^{\prime}+b c^{\prime} \\
0 & c c^{\prime}
\end{array}\right)
$$

where $\boldsymbol{Z}$ acts on $\boldsymbol{Z} /(p)$ in the usual way.

Lemma 1. $T$ is Noetherian. 
Proof. As an abelian group $T$ is finitely-generated.

Lemma 2. (i) If

$$
r\left(\left(\begin{array}{ll}
a & b \\
0 & c
\end{array}\right)\right)=0
$$

then $a \notin(p)$. (ii) Let

$$
x=\left(\begin{array}{ll}
p & 0 \\
0 & 1
\end{array}\right),
$$

then $l(x)=0$ and $r(x) \neq 0$.

Proof. For part (i), suppose $a \in(p)$, then

$$
\left(\begin{array}{ll}
a & b \\
0 & c
\end{array}\right)\left(\begin{array}{ll}
0 & 1 \\
0 & 0
\end{array}\right)=0 .
$$

Part (ii) is clear.

Lemma 3. (i) $Z_{l}(T) \neq 0$.

(ii) $Z_{r}(T)=0$.

(iii) If $t \in T$ and $r(t)=0$, then $l(t)=0$ and $t$ is regular.

Proof. Since $T$ is Noetherian and $l(x)=0, T x$ is an essential left ideal. But

$$
T x \subset l\left(\left(\begin{array}{ll}
0 & 1 \\
0 & 0
\end{array}\right)\right) .
$$

It is well-known that the right singular ideal of a Noetherian ring is nilpotent. But,

$$
N(T)=\left\{\left(\begin{array}{ll}
0 & u \\
0 & 0
\end{array}\right) \mid u \in \boldsymbol{Z} /(p)\right\} .
$$

Thus, if $n \in Z_{r}(T), r(n) \cap J=0$, where

$$
J=\left\{\left(\begin{array}{ll}
0 & 0 \\
0 & j
\end{array}\right) \mid j \in \boldsymbol{Z} /(p)\right\} .
$$

Finally, if $r(t)=0$, then $t T$ is essential, and if $t_{1} t=0$ we have $t_{1} \in Z_{r}(T)$. So $l(t)=0$.

We now note that the subring

$$
T_{1}=\left\{\left(\begin{array}{ll}
a & 0 \\
0 & b
\end{array}\right) \mid a \in \boldsymbol{Z}, b \in \boldsymbol{Z} /(p)\right\}
$$


is isomorphic to $\boldsymbol{Z} \oplus \boldsymbol{Z} /(p)$. All the pieces are now available to construct the examples. The ring $T$ has some amusing properties about which more will be said later.

Let $S$ be the ring of all two-by-two "matrices" of the form

$$
\left(\begin{array}{ll}
t & t^{\prime} \\
0 & t_{1}
\end{array}\right),
$$

$t, t^{\prime} \in T$ and $t_{1} \in T_{1}$. The ring operations are defined as above with $T$ and $T_{1}$ acting on $T$ by ordinary multiplication in $T$.

Lemma 4. $S$ is Noetherian.

Proof. It is clear that $S$ is finitely-generated as an abelian group. Let

$$
y=\left(\begin{array}{ll}
1 & 0 \\
0 & x
\end{array}\right)
$$

where $x$ is, as before, equal to

$$
\left(\begin{array}{ll}
p & 0 \\
0 & 1
\end{array}\right) \in T_{1}
$$

Lemma 5. $y$ is a regular element of $S$.

Proof. We show $l(y)=0$; the other case is similar. Suppose

$$
\left(\begin{array}{ll}
a & b \\
0 & c
\end{array}\right)\left(\begin{array}{ll}
1 & 0 \\
0 & x
\end{array}\right)=0 .
$$

Then, of course, $a=0, b x=0$ forces $b=0$ as $l_{T}(x)=0$ and $c x=0$ yields $c=0$ as $x$ is regular in $T_{1}$.

Lemma 6. If

$$
\left(\begin{array}{ll}
a & b \\
0 & d
\end{array}\right)
$$

is regular in $S$, then a is regular in $T$.

Proof. By Lemma 3, it suffices to show that $r_{T}(a)=0$. If not, there is a $t \neq 0$ such that $a t=0$. But, then

$$
\left(\begin{array}{ll}
a & b \\
0 & d
\end{array}\right)\left(\begin{array}{ll}
t & 0 \\
0 & 0
\end{array}\right)=0
$$

We now can show that $S$ does not satisfy the left Ore condition and, hence, $S$ does not have a left quotient ring. Consider $y$ and the element 


$$
\left(\begin{array}{ll}
0 & \left.\begin{array}{ll}
c & 0 \\
0 & 0
\end{array}\right) \\
0 & 0
\end{array}\right)
$$

where $c \notin(p)$.

THEOREM 1. $S$ does not satisfy the left Ore conaition.

Proof. If the Ore condition held, we would have an equation

where

$$
\left(\begin{array}{ll}
a_{1} & b_{1} \\
0 & d_{1}
\end{array}\right)\left(\begin{array}{ll}
1 & 0 \\
0 & x
\end{array}\right)=\left(\begin{array}{ll}
a & b \\
0 & d
\end{array}\right)\left(\begin{array}{cc}
0\left(\begin{array}{ll}
c & 0 \\
0 & 0
\end{array}\right) \\
0 & 0
\end{array}\right),
$$

$$
\left(\begin{array}{ll}
a & b \\
0 & d
\end{array}\right)
$$

is regular in $S$. By Lemma 6, this means that $a$ is regular in $T$. The above equation forces

$$
b_{1} x=a\left(\begin{array}{ll}
c & 0 \\
0 & 0
\end{array}\right) .
$$

Writing this out fully we obtain

$$
\left(\begin{array}{ll}
e_{1} & f_{1} \\
0 & g_{1}
\end{array}\right)\left(\begin{array}{ll}
p & 0 \\
0 & 1
\end{array}\right)=\left(\begin{array}{ll}
e & f \\
0 & g
\end{array}\right)\left(\begin{array}{ll}
c & 0 \\
0 & 0
\end{array}\right)
$$

Since $a$ is regular, $e \notin(p)$. However, this last relation yields $e_{1} p$ $=e c \neq 0$. But, $e_{1} p \in(p)$ and $e c \notin(p)$ as neither $e$ nor $c$ is, and a contradiction has been reached.

It can be shown that $S$ has a right quotient ring. Let $S^{0}$ be the opposite ring of $S . S^{0}$ is again Noetherian, but $S^{0}$ does not possess a right quotient ring. Hence, it follows that

Theorem 2. $S \oplus S^{0}$ is Noetherian but satisfies neither Ore condition.

Returning to $S$, we find that:

$$
N(S)=\left\{\left(\begin{array}{ll}
n & t \\
0 & 0
\end{array}\right) \mid n \in N(T), t \in T\right) .
$$

Hence, $N(S)^{3}=0$ and $S / N(S) \approx T_{1} \oplus T_{1}$. Therefore, given $s_{1}, s_{2} \in S$ it follows that $\left(s_{1} s_{2}-s_{2} s_{1}\right)^{3}=0 . S$ is, thus, a fairly well-behaved ring; indeed, it is a finitely-generated algebra over $\boldsymbol{Z}$. It also should be noted that the fact that $S$ has a quotient ring on only one side while 
enjoying chain conditions on both is in marked contrast to the semiprime case.

Finally, let us look a little at $T$. The following facts can be proved easily. $T$ has global dimension two ( $T$ is Noetherian so there is no left-right problem), and $T$ has a two-sided quotient ring which is not Artinian. This last fact shows that the result in [2] is, in some sense, best possible. Also, the existence of the element $x$ with $l(x)=0$, but $r(x) \neq 0$ in a Noetherian ring with unit is of some interest.

\section{REFERENCES}

1. A. W. Goldie, Semi-prime rings with maximum condition, Proc. London Math. Soc. (3) 10 (1960), 201-220.

2. L. W. Small, Hereditary rings," Proc. Nat. Acad. Sci. U.S.A. 55 (1966), 25-27.

3. - Orders in Artinian rings, J. Algebra, (to appear 1966).

University of California, Berkeley 